\section{Therapeutic efficacy of low-dose steroid combined with hyaluronidase in ultrasonography-guided intra-articular injections into the shoulder for adhesive capsulitis}

Jong Hyuk Lee ${ }^{1}$, Eun Jung Choi ${ }^{2}$, Seok Cheol Han ${ }^{1}$, Hee Sup Chung ${ }^{1}$, Mi Jung Kwon ${ }^{3}$, Prathap Jayaram ${ }^{4}$, Wonjae Lee ${ }^{1}$, Michael Y. Lee ${ }^{4}$

${ }^{1}$ Department of Physical Medicine and Rehabilitation, Veterans Health Service Medical Center, Seoul; ${ }^{2}$ Department of Rehabilitation Medicine, Seongnam Citizens Medical Center, Seongnam; ${ }^{3}$ Department of Pathology, Hallym University Sacred Heart Hospital, Hallym University College of Medicine, Anyang, Korea; ${ }^{4} \mathrm{H}$. Ben Taub Department of Physical Medicine and Rehabilitation, Baylor College of Medicine, Houston, TX, USA

Purpose: The purpose of this study was to compare the efficacy of low-dose steroid, highdose steroid, and low-dose steroid combined with hyaluronidase with respect to intra-articular injection therapy for adhesive capsulitis (AC) of the shoulder.

Methods: Thirty patients with primary AC in the initial stage were randomly assigned into three groups to receive ultrasound-guided intra-articular injections with $20 \mathrm{mg}$ of triamcinolone acetonide (group $A, n=10$ ), $40 \mathrm{mg}$ of triamcinolone acetonide (group $B, n=10$ ) and $20 \mathrm{mg}$ of triamcinolone acetonide combined with hyaluronidase (group $C, n=10$ ). The outcome measures included a visual analogue scale (VAS), the Shoulder Disability Questionnaire (SDQ), abduction and external rotation range of motion, and intra-sheath fluid (ISF) before treatment and at 2, 4, 8, and 16 weeks after treatment.

Results: Among the 30 patients, one participant in group B dropped out; therefore, a total of 29 patients completed this study and were successfully injected. After the injection, the VAS, SDQ, range of flexion and external rotation, and ISF improved in all groups compared with the preinjection status, regardless of treatment or time point. In the comparison between groups, the SDQ and ISF showed significantly greater improvements in groups B and $C$ than in group A.

Conclusion: The therapeutic efficacy of combined low-dose corticosteroid and hyaluronidase is superior to that of low-dose corticosteroid and equivalent to that of high-dose corticosteroid in early $A C$.

Keywords: Adhesive capsulitis; Intra-articular injections; Steroids; Hyaluronidase; Ultrasonography

e-ultrasonography.org

Ultrasonography 40(4), October 2021

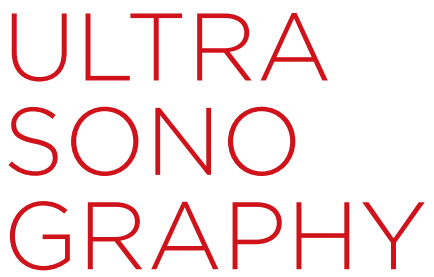

\section{ORIGINAL ARTICLE}

https://doi.org/10.14366/usg.20199 pISSN: 2288-5919 • elSSN: 2288-5943

Ultrasonography 2021;40:555-564

Received: December 11, 2020

Revised: February 16, 2021

Accepted: February 22, 2021

Correspondence to:

Wonjae Lee, MD, PhD, Department of Physical Medicine and Rehabilitation, Veterans Health Service Medical Center, 53 Jinhwangdo-ro 61-gil, Gangdong-gu, Seoul 05368, Korea

Tel. +82-2-2225-1397

Fax. +82-2-2225-1391

E-mail: braddom@nate.com

Michael Y. Lee, MD, MHA, H. Ben Taub Department of Physical Medicine and Rehabilitation, Baylor College of Medicine, 7200 Cambridge Street, Houston, TX 77030, USA

Tel. +1-713-798-2033

Fax. +1-713-798-7259

E-mail: Michael.Lee@bcm.edu

This is an Open Access article distributed under the terms of the Creative Commons Attribution NonCommercial License (http://creativecommons.org/ licenses/by-nc/4.0/) which permits unrestricted noncommercial use, distribution, and reproduction in any medium, provided the original work is properly cited.

Copyright (? 2021 Korean Society of Ultrasound in Medicine (KSUM)

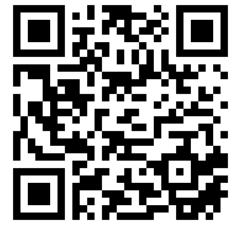

How to cite this article:

Lee JH, Choi EJ, Han SC, Chung HS, Kwon $M J$, Jayaram $P$, et al. Therapeutic efficacy of low-dose steroid combined with hyaluronidase in ultrasonography-guided intra-articular injections into the shoulder for adhesive capsulitis. Ultrasonography. 2021 0ct;40(4):555-564. 


\section{Introduction}

Adhesive capsulitis, also known as frozen shoulder or arthrofibrosis, is an orthopedic condition defined as a painful, progressive restriction of both the active and passive ranges of shoulder motion [1]. It is a common condition, affecting $2 \%-5 \%$ of the general population. The factors associated with primary adhesive capsulitis include female sex, trauma, age older than 40 years, diabetes, prolonged immobility, thyroid disease, stroke, myocardial infarcts, and autoimmune disease $[2,3]$. The underlying mechanism of primary adhesive capsulitis is thought to be chronic degenerative inflammation across the capsular synovial layer of the glenohumeral joint, which produces capsular thickening, fibrosis, and adherence of the capsule to itself and to the anatomic neck of the humerus, leading to pain, stiffness, and dysfunction [4]. Although almost half of patients with adhesive capsulitis experience restricted shoulder motion lasting up to 7 years [5], self-limiting resolution within $1-3$ years has also been reported [6], implying that there is still therapeutic room for a favorable prognosis in adhesive capsulitis.

The natural progress of adhesive capsulitis involves three stages: (1) increasing pain and stiffness, (2) a steady-state period, and (3) a spontaneous recovery period. Particularly in the initial stage, increasingly severe shoulder pain and stiffness, lasting for 2-9 months, and accompanying synovitis lead to limitations in abduction and external rotation range of motion $[7,8]$. Since the goal of adhesive capsulitis treatment is to restore the shoulder to a painless and functional joint [9], it is important to reduce the inflammatory reaction in the initial pain stage, which causes reactive fibrosis of the glenohumeral joint capsule. Intra-articular glenohumeral steroid injections have become widely utilized in the treatment of adhesive capsulitis among various non-operative management strategies $[10,11]$, because this method has been observed to offer faster and superior improvement of symptoms compared to oral steroid treatment or physical therapy $[12,13]$. The efficacy of intra-articular steroid injections is also related to its anti-inflammatory properties that relieve pain and restore shoulder range of motion and function [12].

Hyaluronidases are a family of enzymes that catalyze the degradation of hyaluronic acid. By catalyzing the hydrolysis of hyaluronan, a constituent of the extracellular matrix, hyaluronidase lowers the viscosity of hyaluronan, thereby increasing the tissue permeability of injected solutes $[14,15]$. Accordingly, hyaluronidase is used in medicine in conjunction with other drugs, including steroids, to speed their dispersion and delivery $[14,15]$. There is growing evidence that using a steroid in combination with hyaluronidase in shoulder joints or spinal epidural spaces both maximizes the anti-inflammatory properties of the steroid and minimizes the side effects of steroid therapy [16-18]. A few studies have reported that the combined injection of a steroid and hyaluronidase showed an additive effect with regard to improvements in pain and shoulder function in patients with periarticular shoulder disorders $[16,18]$. However, those studies were not specifically focused on adhesive capsulitis. There is still a lack of prospective randomized controlled studies to verify the effectiveness of administering hyaluronidase in intra-articular steroid injections for adhesive capsulitis by simultaneous monitoring of symptomatic, anthropometric, and imaging changes.

Therefore, we hypothesized that the co-administration of hyaluronidase could reduce the total amount of steroids needed to treat adhesive capsulitis of the shoulder. This study aimed to verify the therapeutic efficacy of a low-dose steroid combined with hyaluronidase as compared with low-dose and high-dose steroid formulations in ultrasound-guided intra-articular injections for the treatment of adhesive capsulitis.

\section{Materials and Methods}

\section{Compliance with Ethical Standards}

The present study was approved by the ethics committee of Veterans Health Service Medical Center in Korea (IRB No. 2017-03-011001), and all participants gave informed consent after receiving an explanation about the purpose, methods, and policy regarding the personal information collected from this study.

\section{Participants and Study Design}

Between May 2017 and March 2019, a total of 1,035 consecutive patients with complaints of shoulder pain who visited the outpatient clinic of our institution were screened for eligibility. Those who met the following inclusion criteria were considered to have inflammatory synovitis of initial-stage adhesive capsulitis [19] (Fig. 1): (1) normal findings on simple $X$-rays of the shoulder, but limitations in the passive range of motion on physical examination; and (2) increased intra-sheath fluid (ISF) sufficient to encircle the long head of the biceps tendon within the upper portion of the bicipital groove of the humerus. The upper portion of the bicipital groove of the humerus corresponds to the synovial extension that anteriorly communicates with the glenohumeral joint through the rotator cuff interval. An obvious increase in ISF in the upper portion of the bicipital groove of the humerus without any structural abnormalities indicates the state of inflammatory synovitis [20]. The exclusion criteria were as follows: (1) ultrasonographic diagnosis of other concomitant fundamental abnormalities causing shoulder pain such as rotator cuff tear, bicipital tendon rupture, calcific tendinopathy, and subacromial-subdeltoid bursitis; (2) a history of shoulder injury; 


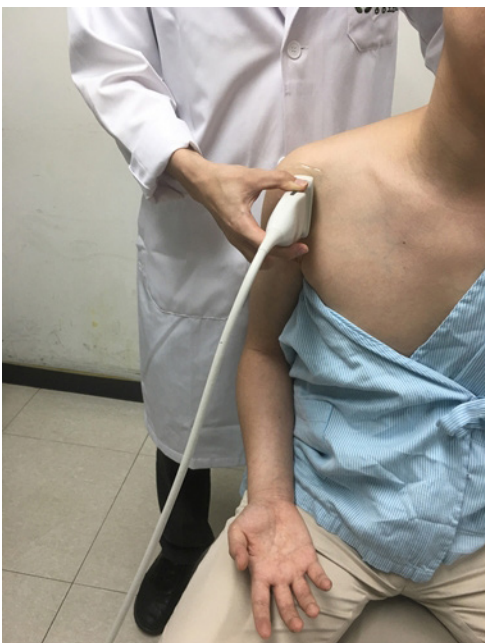

A

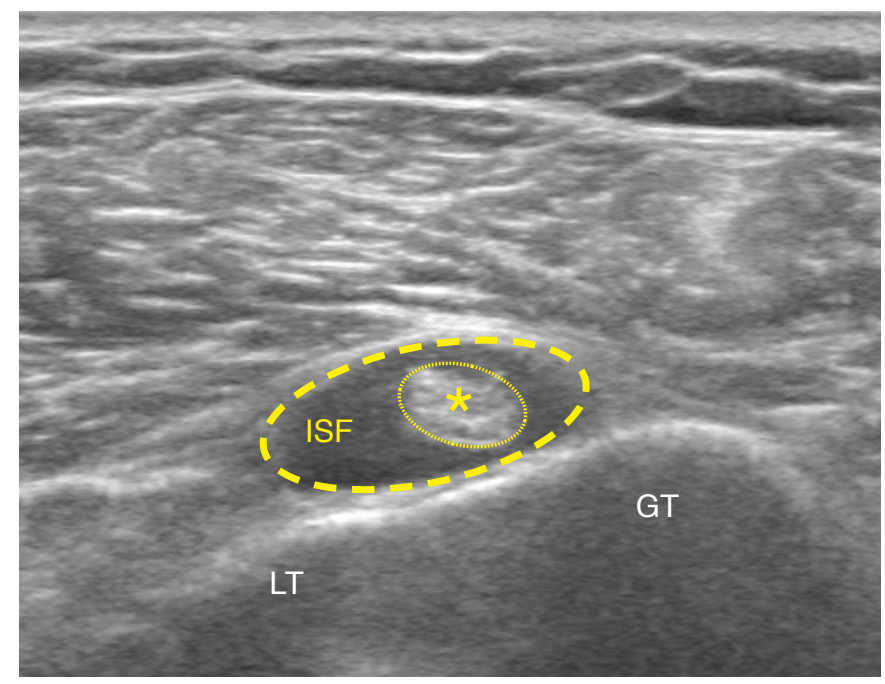

C

(3) a history of more than 1 year of conservative treatment for chronic shoulder pain; (4) corticosteroid or hyaluronidase injections within the prior 6 months; (5) hemiplegic shoulder; (6) self-reported history consistent with scapula fracture or disarticulation; (7) an ipsilateral cervical herniated intervertebral disc or brachial plexus injury; (8) diabetes mellitus refractory to insulin therapy or glycated hemoglobin levels greater than $6.5 \%$; and (9) refusal to participate in this study.

Overall, 1,005 patients did not meet the inclusion criteria. Finally, 30 patients with primary adhesive capsulitis were included, and this study was conducted as a double-blind randomized controlled trial. A schematic flow diagram of the study is shown in Fig. 2. The eligible patients $(n=30)$ were randomized into three groups: A, B, and C. Randomization was performed by letting patients choose between three sealed and opaque envelopes; each of
Fig. 1. Ultrasonographic evaluation of the upper part of the bicipital groove of the humerus.

A. Scanning of the right shoulder in the longitudinal plane is shown. B. Identification of the hypoechoic fluid enveloping the normal echotexture of the long head of the biceps tendon (asterisk) along the bicipital groove, which starts from the upper part of the rotator cuff interval $(\mathrm{RCl})$ and does not extend beyond the lower part. The solid line indicates the cut-off point of the most inflated level for the axial scanning of C. C. The hypoechoic fluid around the long head of the biceps tendon (asterisk) between the lesser tuberosity (LT) and the greater tuberosity (GT) of the humerus. Intra-sheath fluid (ISF) was calculated by subtracting the area of the inner circle (dotted line) from that of the outer circle (dashed line).

these envelopes contained allocation to one of the three treatment groups, giving every patient the same allocation opportunity.

\section{Sample Size Calculation}

For the sample size calculation, assumptions about the primary outcome values and variance were based on a previous study on adhesive capsulitis by Choi et al. [18]. Assuming that analysis of variance (ANOVA) would be conducted among the three groups with standard deviations of 2 points for both within-group and betweengroup differences, our power analysis indicated that a minimum of eight patients per group was needed (power of $90 \%, a=0.05$ ). To account for a possible loss to follow-up of up to $20 \%$, a minimum of 10 patients per group needed to be enrolled in this study. 


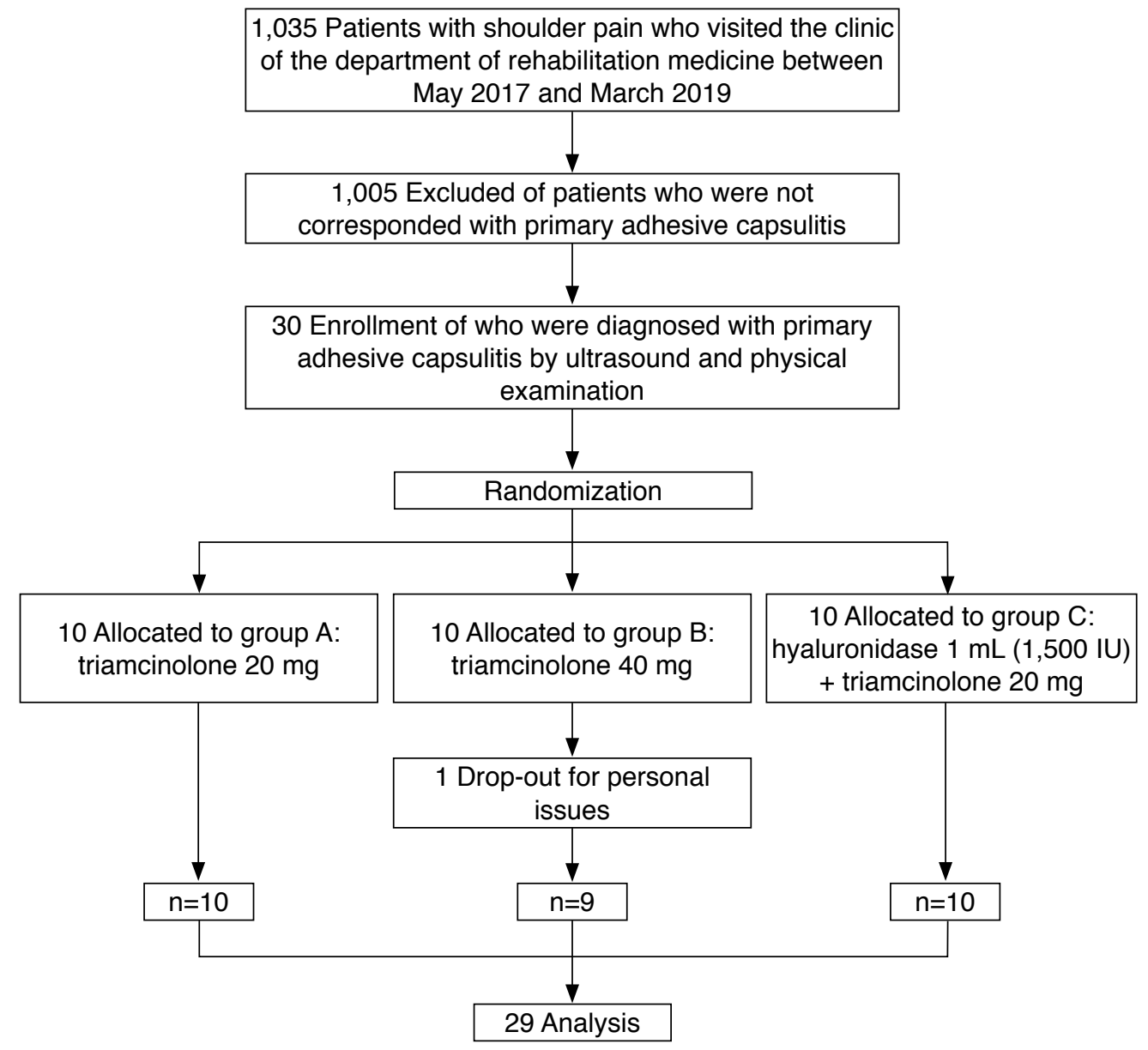

Fig. 2. CONSORT (Consolidated Standards of Reporting Trials) diagram of the study flow.

\section{Intra-articular Injections}

All patients underwent a physical examination, diagnostic shoulder radiographs, and ultrasonography to confirm the lesion [21]. After confirmation of the initial stage of adhesive capsulitis by ultrasonography, one investigator (a medical doctor who has been certified in the field of musculoskeletal ultrasound by the Korean Society of Ultrasound in Medicine) performed ultrasound-guided intra-articular injections into the shoulders of patients. While asking the subject to hold the contralateral shoulder with the hand of the symptomatic side in the sitting position, the ultrasound transducer was placed on the posterior surface of the shoulder in the longitudinal plane to identify the posterior glenohumeral joint, bony glenoid, humeral head, and infraspinatus muscle [22]. Then, after sterilizing the area to be injected, the needle ( $23 \mathrm{G}$, $6 \mathrm{~cm}$, KOVAX-NEEDLE, Korea Vaccine Co., Seoul, Korea) was advanced to the target point between the humeral head cartilage and the infraspinatus tendon using the ultrasound-guided in-plane technique. To ensure the injection into the glenohumeral joint, the Doppler mode was used to confirm the widening of the space between the humeral cartilage and the innermost synovial line of the infraspinatus muscle by the injected solution (Fig. 3).

All patients received one type of intra-articular injection according to their group. Group A $(n=10)$ received $20 \mathrm{mg}(0.5 \mathrm{~mL})$ of triamcinolone acetonide mixed with $2 \mathrm{~mL}$ of $2 \%$ lidocaine and $7.5 \mathrm{~mL}$ of normal saline. Group B ( $\mathrm{n}=10)$ received $40 \mathrm{mg}(1 \mathrm{~mL})$ of triamcinolone acetonide mixed with $2 \mathrm{~mL}$ of $2 \%$ lidocaine and 7.0 $\mathrm{mL}$ of normal saline. Group $\mathrm{C}(\mathrm{n}=10)$ received $20 \mathrm{mg}(0.5 \mathrm{~mL})$ of triamcinolone acetonide and $1 \mathrm{~mL}$ of hyaluronidase (Hirax Inj MBI KOREA Pharmaceutical, Cheongju, Korea) mixed with $2 \mathrm{~mL}$ of $2 \%$ lidocaine and $6.5 \mathrm{~mL}$ of normal saline. The composition of $10 \mathrm{~mL}$ of $0.4 \%$ lidocaine was the same in all the injected solutions. After the injection, the participants were educated on home-based basic exercises involving stretching and pendulum circumduction. No additional medication or physical therapies were administered until completion of this study.

\section{Efficacy Evaluation}

The patients were evaluated at five time intervals: before injection and at 2, 4, 8, and 16 weeks after the injection. First, subjects were asked to describe their symptoms using a visual analogue scale (VAS) 


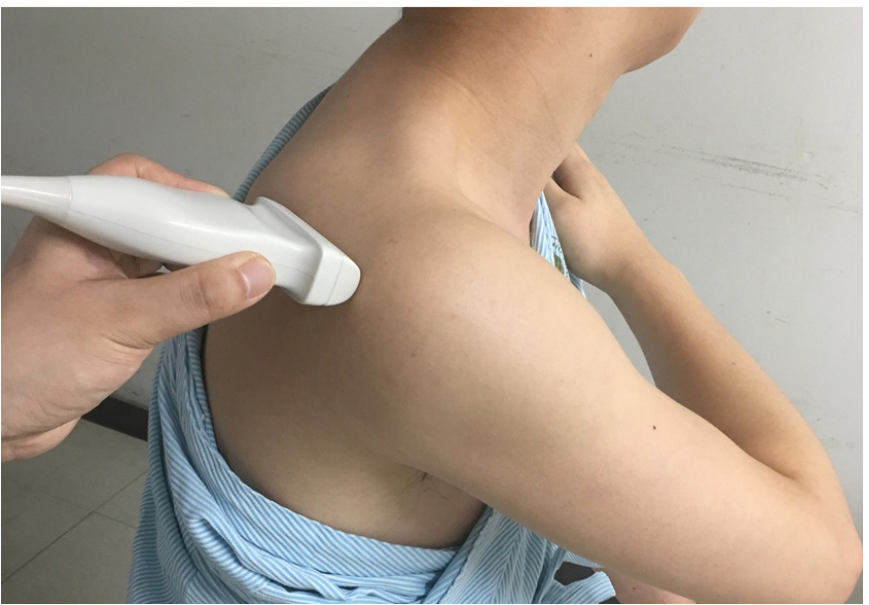

A

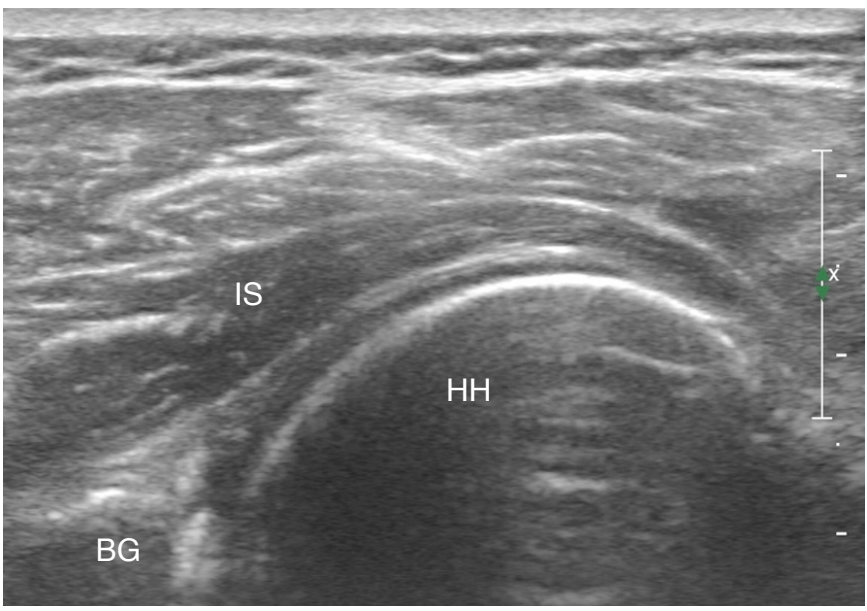

C

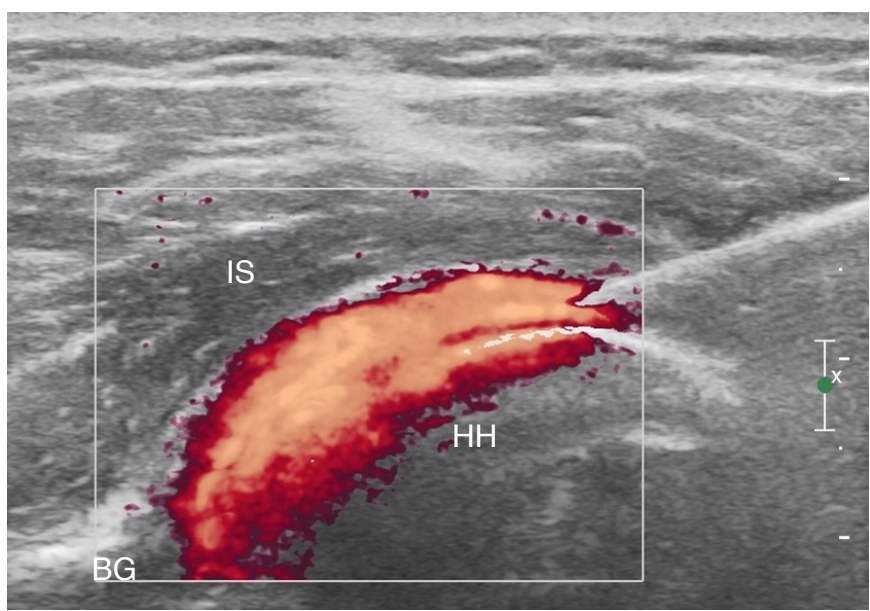

E

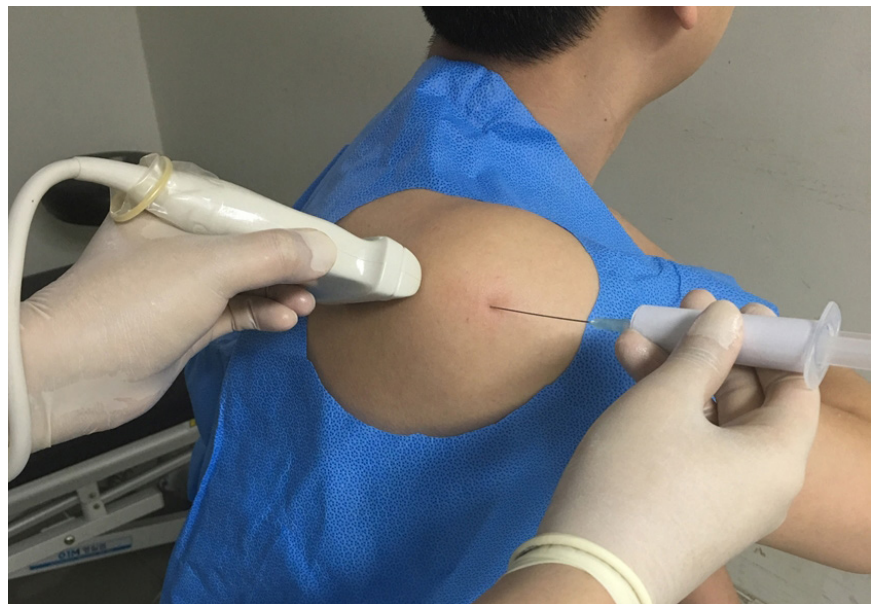

B

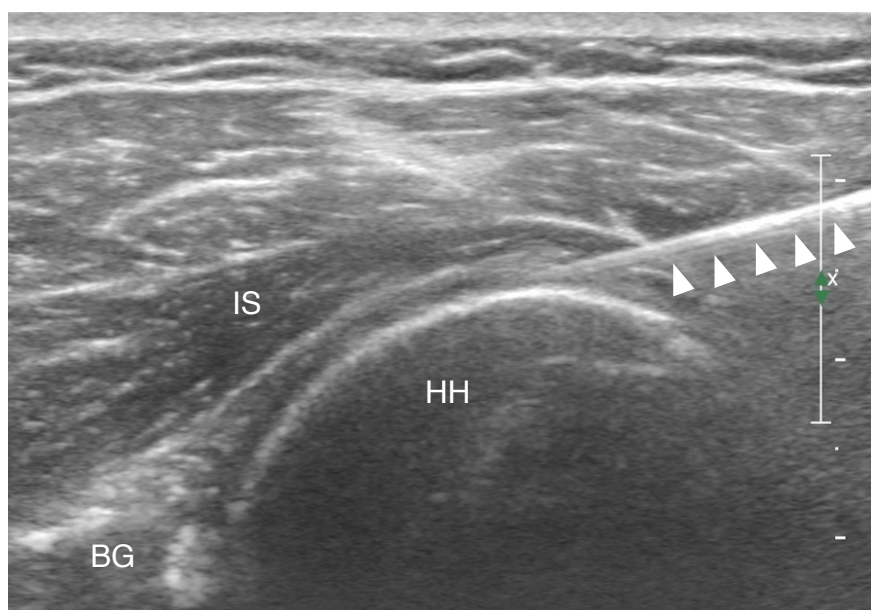

D

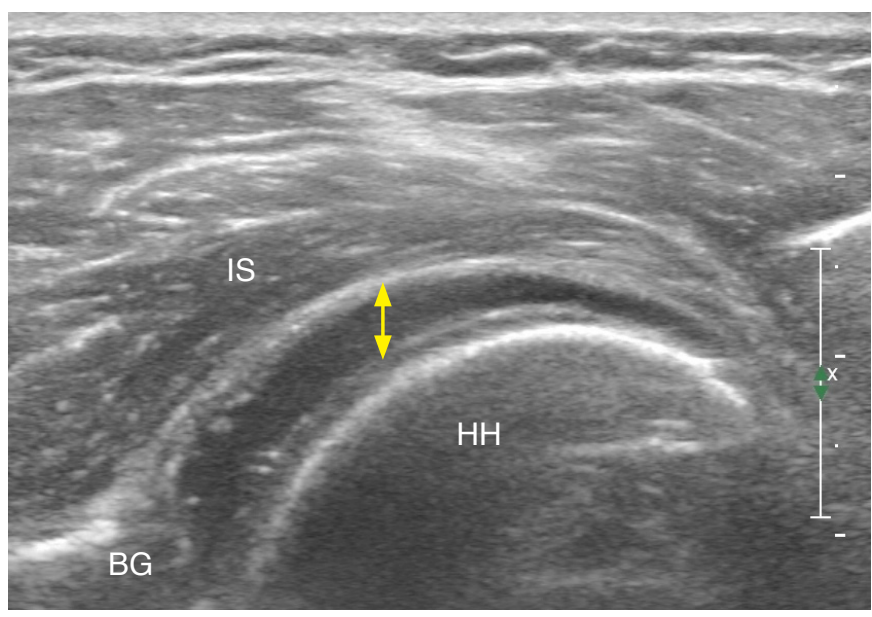

$\mathrm{F}$

Fig. 3. Ultrasound-guided intra-articular injection.

A. Scanning of posterior shoulder is shown. B. Injection after preparation is shown. C. Transverse scanning image is shown. D. Needle advanced state is shown. E. Injection streaming, which is visible in the Doppler mode, into the joint space between the bony glenoid (BG) and the humeral head $(\mathrm{HH})$ is shown. F. Gap widening (up-down arrow) between the humeral cartilage and the innermost synovial line of the infraspinatus muscle after injection is shown. IS, infraspinatus. 
and the Shoulder Disability Questionnaire (SDQ). The VAS was used to rate the average degree of shoulder pain for 24 hours before the evaluation (from 0 to 10 points). The SDQ, the validity and reliability of which has been confirmed [23], was used to evaluate the selfreported functional status in patients with shoulder disorders, The SDQ is composed of 16 questions reflecting pain during various movements related with activities of daily living, with scores ranging from 0 (no disability) to 16 (greatest possible disability). Second, the abduction and external rotation range of motion of the symptomatic shoulder were evaluated. The anthropometric variables were checked with an inclinometer, as a passive maneuver, in the supine position. Each range was measured three times, and the average value was recorded. Lastly, ISF was measured through an ultrasonographic evaluation to determine any changes on imaging.

\section{Statistical Analysis}

All the statistical analyses were performed using SPSS version 19.0 for Windows (IBM Corp., Armonk, NY, USA). Basic variables and pre-injection parameters among the three groups were tested for significance using ANOVA or the chi-square test, as appropriate. Repeated-measures ANOVA were used for the statistical analysis of changes in the VAS, SDQ, range of motion, and ISF in the three groups before the injection and at 2, 4, 8, and 16 weeks after treatment. For between-group comparisons, post-hoc tests were conducted using the Tukey method. All cases where the P-value was less than 0.05 were considered statistically significant.

\section{Results}

Thirty subjects were randomly assigned to groups $A, B$, and $C$, with 10 subjects in each group. Among these 30 patients with primary adhesive capsulitis of the shoulder, only one patient (in group B) was lost to follow-up for personal reasons. Finally, a total of 29 patients completed this study. The baseline characteristics and clinical profiles of those 29 patients were investigated, and the comparisons among groups $A, B$, and $C$ are shown in Table 1. There were no significant differences in age, sex, height, weight, affected side, the dominant side, and symptom duration among the groups.

\section{Comparisons of Symptomatic, Anthropometric, and Imaging Changes in Each Group}

Variable values for the VAS, SDQ, shoulder abduction and external rotation, and ISF were observed during follow-up in the three groups before and after the injections. The changes in the VAS, SDQ, shoulder abduction and external rotation, and ISF were statistically analyzed by repeated-measures ANOVA in the three treatment groups for 16 weeks. The scores of group C showed trends toward
Table 1. Baseline characteristics and clinical profiles of each study group (low-dose steroid [group A], high-dose steroid [group B], and combined low-dose steroid and hyaluronidase [group C])

\begin{tabular}{lcccc}
\hline \multicolumn{1}{c}{ Variable } & $\begin{array}{c}\text { Group A } \\
(\mathrm{n}=10)\end{array}$ & $\begin{array}{c}\text { Group B } \\
(\mathrm{n}=9)\end{array}$ & $\begin{array}{c}\text { Group C } \\
(\mathrm{n}=10)\end{array}$ & P-value \\
\hline Age (year) & $71.30 \pm 9.37$ & $68.56 \pm 6.97$ & $74.4 \pm 4.93$ & 0.239 \\
Sex (female/male) & $4 / 6$ & $5 / 4$ & $3 / 7$ & 0.525 \\
Height (cm) & $166.00 \pm 9.07$ & $167.22 \pm 8.32$ & $165.9 \pm 7.91$ & 0.932 \\
$\begin{array}{l}\text { Weight (kg) } \\
\text { Affected side }\end{array}$ & $64.40 \pm 12.92$ & $66.11 \pm 12.62$ & $67.10 \pm 11.89$ & 0.888 \\
(right/left) & $6 / 4$ & $7 / 2$ & $8 / 2$ & 0.552 \\
$\begin{array}{l}\text { Dominant side } \\
\text { (right/left) }\end{array}$ & $8 / 2$ & $8 / 1$ & $7 / 3$ & 0.596 \\
$\begin{array}{l}\text { Duration of } \\
\text { symptoms (month) }\end{array}$ & $4.70 \pm 1.64$ & $5.00 \pm 1.94$ & $4.30 \pm 1.70$ & 0.687 \\
\hline
\end{tabular}

P-value for comparison between groups by analysis of variance or the chi-square test.

the lowest levels among the three groups. The distributions of the VAS, SDQ, shoulder abduction and external rotation ranges, and ISF values of each group according to the follow-up time of 16 weeks are shown in Table 2.

Compared with pre-injection, both the VAS and SDQ symptom scores significantly decreased during the 16-week follow-up period within each group (both $\mathrm{P}<0.001$ ), indicating significant pain relief after treatment within each group. In addition, the anthropometric values of both shoulder abduction and external rotation range of motion significantly increased $(\mathrm{P}=0.018$ and $\mathrm{P}=0.004$, respectively), indicating meaningful improvement in the range of motion after treatment within each group. Moreover, the extent of ISF at the affected shoulder also significantly decreased after treatment within each group $(P<0.001)$, which confirmed improvements in inflammatory synovitis through imaging measurements over time.

Therefore, in the evaluation of the therapeutic effect, the statistical analysis of repeated-measures ANOVA showed a significant time effect for the VAS, SDQ, abduction range of motion, external rotation range of motion, and ISF. Taken together, our results showed that the co-administration of hyaluronidase and intra-articular steroid injection itself, regardless of the steroid dose, was effective in patients in the initial phase of adhesive capsulitis.

\section{Comparisons of the Symptomatic, Anthropometric, and Imaging Changes between Groups}

We further evaluated the effectiveness of the co-administration of hyaluronidase and steroid therapy to adjust the time effect. For the SDQ, repeated-measures ANOVA showed both a significant time effect $(F=24.21, P<0.001)$ in each group (as mentioned above) and a group effect $(F=5.39, P=0.011)$ according to the three groups. 
Table 2. Repeated-measures analysis of variance (ANOVA) results of the therapeutic effect with regard to VAS, SDQ, shoulder AB, shoulder ER, and ISF in the treatment groups (low-dose steroid [group A], high-dose steroid [group B], and combined low-dose steroid and hyaluronidase [group C])

\begin{tabular}{|c|c|c|c|c|c|c|}
\hline \multirow{2}{*}{ Variable } & \multirow{2}{*}{ Pre-injection } & \multicolumn{4}{|c|}{ Post-injection } & \multirow{2}{*}{ P-value ${ }^{a)}$} \\
\hline & & 2 weeks & 4 weeks & 8 weeks & 16 weeks & \\
\hline VAS & $P=0.530$ & & & & & $<0.001^{\text {a) }}$ \\
\hline Group B (points) & $4.89 \pm 1.05$ & $2.44 \pm 0.73$ & $2.89 \pm 1.27$ & $2.78 \pm 1.40$ & $2.67 \pm 1.32$ & \\
\hline Group C (points) & $5.70 \pm 2.06$ & $3.80 \pm 1.69$ & $3.30 \pm 1.57$ & $3.00 \pm 1.63$ & $2.70 \pm 2.00$ & \\
\hline Group A (points) & $11.10 \pm 1.85$ & $9.60 \pm 3.27$ & $8.80 \pm 3.33$ & $9.10 \pm 2.64$ & $9.10 \pm 1.91$ & \\
\hline Group B (points) & $10.78 \pm 2.59$ & $7.22 \pm 2.11$ & $5.78 \pm 1.09$ & $5.78 \pm 1.20$ & $5.33 \pm 2.92$ & \\
\hline Group C (points) & $10.40 \pm 2.01$ & $7.70 \pm 2.16$ & $6.70 \pm 2.67$ & $6.30 \pm 2.63$ & $5.90 \pm 2.73$ & \\
\hline Shoulder AB & $P=0.550$ & & & & & $0.018^{a)}$ \\
\hline Group A $\left(^{\circ}\right)$ & $120.80 \pm 14.80$ & $126.30 \pm 11.80$ & $125.30 \pm 14.05$ & $126.10 \pm 13.47$ & $126.30 \pm 17.64$ & \\
\hline Group A $\left(^{\circ}\right)$ & $55.30 \pm 10.83$ & $58.30 \pm 10.00$ & $58.20 \pm 9.66$ & $57.80 \pm 8.19$ & $57.20 \pm 12.39$ & \\
\hline Group B $\left(^{\circ}\right)$ & $57.33 \pm 16.61$ & $66.22 \pm 5.72$ & $67.44 \pm 7.63$ & $68.78 \pm 9.55$ & $66.89 \pm 11.17$ & \\
\hline Group $C\left(^{\circ}\right)$ & $57.10 \pm 15.72$ & $62.70 \pm 12.68$ & $66.40 \pm 11.76$ & $68.00 \pm 8.26$ & $65.50 \pm 11.78$ & \\
\hline ISF & $P=0.013^{b)}$ & & & & & $<0.001^{\mathrm{a})}$ \\
\hline Group A $\left(\mathrm{mm}^{2}\right)$ & $30.85 \pm 6.88$ & $25.02 \pm 5.39$ & $29.61 \pm 8.56$ & $30.50 \pm 8.08$ & $30.95 \pm 7.08$ & \\
\hline Group B (mm²) & $32.09 \pm 18.51$ & $14.39 \pm 8.07$ & $16.39 \pm 8.37$ & $18.38 \pm 8.39$ & $22.31 \pm 10.07$ & \\
\hline Group C $\left(\mathrm{mm}^{2}\right)$ & $27.82 \pm 7.18$ & $15.24 \pm 3.17$ & $16.41 \pm 3.63$ & $18.99 \pm 4.51$ & $21.58 \pm 5.46$ & \\
\hline
\end{tabular}

Values are presented as mean \pm SD.

VAS, visual analogue scale; SDQ, shoulder disability questionnaire; $A B$, abduction; ER, external rotation; ISF, intra-sheath fluid.

${ }^{\text {a) }} \mathrm{P}<0.05$ for the within-group comparison according to time by repeated-measures ANOVA. ${ }^{\text {b) }} \mathrm{P}<0.05$ for the between-group comparison by repeated-measures ANOVA.

In addition, there was no time and group interaction for the SDQ $(\mathrm{F}=1.206, \mathrm{P}=0.316)$. Therefore, the results demonstrate a significant difference in the degree of improvement in the SDQ across the three groups, which was independent of the changes in the SDQ across time in each group during the 16-week follow-up.

However, there was no difference in the VAS change between the groups ( $P=0.530)$. These results indicate that the levels of pain relief with movement after treatment were significantly different among the three groups, but there no difference in pain at rest among the three groups. The changes in ISF also showed both a significant time effect $(\mathrm{F}=31.43, \mathrm{P}<0.001)$ and a group effect $(F=5.14, P=0.013)$. These effects did not represent a time and group interaction ( $F=1.941, P=0.075)$. These findings of statistical significance indicate that the patterns of the changes significantly differed among the three groups, independent of the results of the within-group comparison.

As for range of motion, the differences in shoulder abduction and external rotation ranges among the three groups did not achieve statistical significance $(\mathrm{P}=0.550$ and $\mathrm{P}=0.082$, respectively). However, the external rotation range of motion in groups $B$ and $C$ tended to be higher than that of group A.

In the post-hoc test, the SDQ and ISF scores in groups B and C showed significantly lower levels for pain and inflammatory synovitis than those of group A (Fig. 4), which indicates that the pain relief and anti-inflammatory effects of the combination of triamcinolone acetonide $(20 \mathrm{mg})$ and hyaluronidase $(1 \mathrm{~mL})$ were equivalent to those of triamcinolone acetonide $(40 \mathrm{mg})$. In addition, the pain relief and anti-inflammatory effects of the combination of triamcinolone acetonide $(20 \mathrm{mg})$ and hyaluronidase $(1 \mathrm{~mL})$ or triamcinolone acetonide $(40 \mathrm{mg})$ alone were superior to those of triamcinolone acetonide $(20 \mathrm{mg})$.

\section{Complications}

Every subject was successfully managed using an intra-articular steroid injection for their symptomatic shoulder joint. All intraarticular injections guided by ultrasonography were accurately 


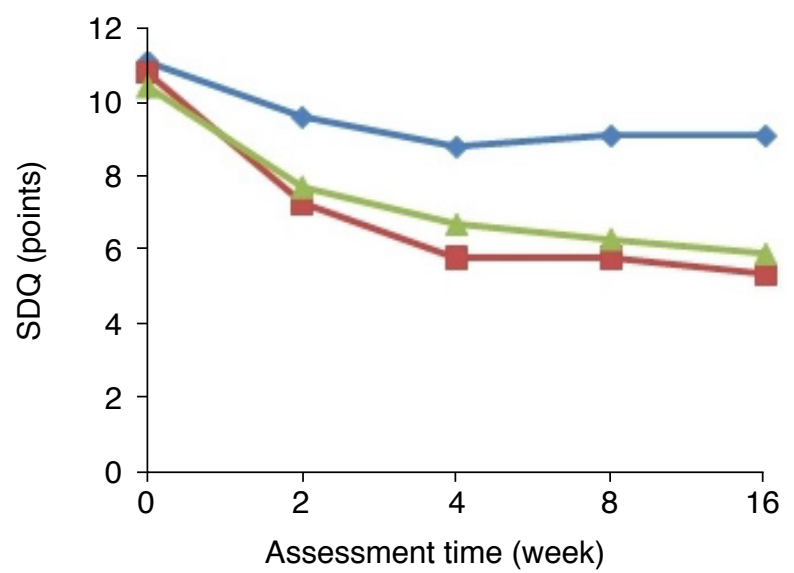

A

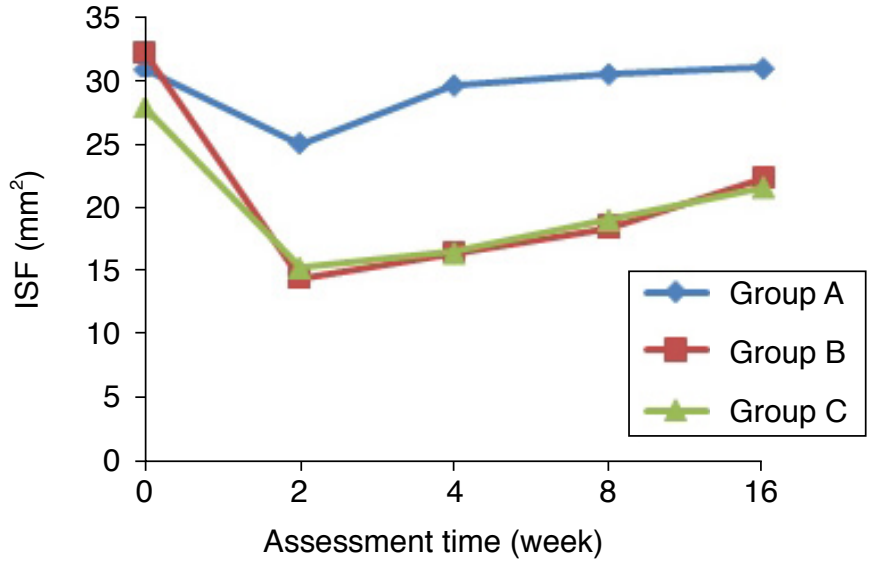

B

Fig. 4. Between-group comparisons.

A, B. Repeated-measures analysis of variance results demonstrate statistically significant differences in the therapeutic effect based on the Shoulder Disability Questionnaire (SDQ) (A) as a symptom score and the intra-sheath fluid (ISF) (B) as an imaging measurement of inflammation according to the treatment groups: low-dose steroid (group A), high-dose steroid (group B), and combined low-dose steroid and hyaluronidase (group C).

targeted, which was confirmed by the injection streaming into the joint space between the bony glenoid and the humeral head on Doppler imaging, in addition to widening of the gap between the humeral cartilage and the innermost synovial line of the infraspinatus muscle after the injection. All interventions were successful the first time, and no acute side effects associated with the procedure were observed. No chronic side effects related to the procedure and the drugs administered were identified at the time of study completion.

\section{Discussion}

Intra-articular combined steroid and hyaluronidase injections have garnered attention as an anticipated treatment for primary adhesive capsulitis, although the evidence of its therapeutic outcomes in clinical trials is scarce. Through a randomized controlled trial, the present study demonstrates that the co-administration of hyaluronidase and steroid therapy via intra-articular injection conferred therapeutic effects in terms of pain relief and antiinflammatory response that were equivalent to those of high-dose steroid monotherapy and superior to those of low-dose steroid monotherapy in the treatment of early adhesive capsulitis. Moreover, patients in all three groups demonstrated improvements in shoulder mobility during the follow-up period, although the betweengroup differences were not statistically significantly different. These benefits were observed to be sustained over 16 weeks beyond the short-term follow-up times of 2, 4, and 8 weeks after the injection. Interestingly, we noted significant changes in the SDQ but not in the VAS, indicating remarkable pain relief during daily activities but not at rest. The addition of hyaluronidase may potentiate the effect of the steroid more efficiently, followed by a substantial reduction in synovial inflammation and symptomatic pain during movement [14]. In this context, the similar trend of pain relief and anti-inflammatory effects between combined hyaluronidase with low-dose steroid therapy and high-dose steroid monotherapy may have occurred because hyaluronidase enhances the permeability of the lowdose steroid to the synovium, the main focus of primary adhesive capsulitis, making it as effective as a high-dose steroid [24].

Our findings confirmed the additive effect of hyaluronidase in low-dose steroid therapy with regard to improvements in pain and inflammatory response in patients with adhesive capsulitis, which is of great clinical importance for the patients who cannot endure high-dose steroid therapy. Unfortunately, the use of steroid injections can cause potentially serious localized side effects associated with tendon rupture, subcutaneous atrophy, and articular cartilage changes, as well as systemic effects such as osteoporosis and hyperglycemia $[9,12,25]$. Adverse effects are more commonly reported in women, including facial flushing and irregular menstrual bleeding $[9,25]$. Of note, hyperglycemia can be fatal because patients with diabetes account for up to $20 \%$ of adhesive capsulitis patients [26]; therefore, steroid injections in patients with subclinical diabetes who have adhesive capsulitis carry a significant risk. In particular, patients with diabetes have been reported to have a high morbidity rate of adhesive capsulitis [27]. Although conservative treatment including steroid injection therapy shows little effect in patients with diabetes [28], controversy persists regarding whether localized steroid injections cause systemic side effects $[26,29]$. The combination of hyaluronidase and low-dose steroid may be the best 
therapeutic option for patients with uncontrolled diabetes who have adhesive capsulitis to reduce the potential risk of adverse systemic effects.

Meanwhile, Kim et al. [30] reported no significant difference in the treatment effect according to the dose of steroid therapy in adhesive capsulitis. Another study showed that low-dose steroid was sufficient to elicit symptom relief in adhesive capsulitis [31]. These discrepancies in findings may be due to differences in ultrasonographic methods and the protocols used to confirm a successfully targeted injection. We thoroughly identified both the injection streaming into the joint space by Doppler imaging and the gap widening between humeral cartilage and the innermost synovial line of the infraspinatus muscle after injection in order to verify the success of each injection. These adhesive capsulitis treatment protocols were not used in the previous studies $[30,31]$.

We randomized 30 patients after the extensive evaluation of 1,035 patients into three groups based on intra-articular injection solutes under ultrasonographic guidance. All three groups with early adhesive capsulitis showed significantly decreased pain, movement restriction, and inflammatory synovitis after treatment compared to pre-injection, implying that accurately targeted early interventions may be clinically relevant in the treatment of early adhesive capsulitis. These results were consistent with those of the previous study, thereby emphasizing the importance of early intraarticular injections for a favorable prognosis in the management of adhesive capsulitis [4]. In the present study, the ultrasonographic guidance during intra-articular injections provided the diagnostic and therapeutic feedback needed to achieve an accurately targeted injection into only the affected areas and not other critical structures (i.e., vessels) and to avoid any serious complications and systemic adverse effects. A recent systematic review of shoulder joint disease also highlighted the increased accuracy and decreased complexity of image-guided injections as compared with blind injections [32], which may be attributable to the advantages of the real-time application of ultrasonography and simultaneous feedback through physician-patient communication [33]. Likewise, we did not have any injection-related complications throughout the study. Despite some claims that ultrasound-guided injections are inferior to blind injections without image guidance $[34,35]$, our trial supports the positive patient-relevant outcomes and safety of ultrasonographic guidance during intra-articular injections in adhesive capsulitis.

There are some limitations in the current study. First, this study was conducted with a small number of patients. Second, we did not have a sufficiently long-term follow-up duration for the evaluation of treatment effects throughout the entire period of adhesive capsulitis. Lastly, there was a lack of enrollment of patients with diabetes who had adhesive capsulitis to validate the treatment outcomes of combined hyaluronidase and low-dose steroid therapy in this highrisk patient population. Further research on a larger scale is needed to determine the effect of combined injection therapy in the late period of adhesive capsulitis, as well as its safety in patients with diabetes.

In conclusion, the therapeutic effects of intra-articular combined hyaluronidase and low-dose steroid injection therapy were found to be equivalent to those of high-dose steroid monotherapy and superior to those of low-dose steroid monotherapy. The addition of hyaluronidase to low-dose steroid therapy resulted in significant clinical improvement in the treatment of early adhesive capsulitis.

ORCID: Jong Hyuk Lee: https://orcid.org/0000-0001-6484-9423; Eun Jung Choi: https:// orcid.org/0000-0001-7894-1730; Seok Cheol Han: https://orcid.org/0000-0003-16305194; Hee Sup Chung: https://orcid.org/0000-0002-1879-502X; Mi Jung Kwon: https:// orcid.org/0000-0002-2441-0448; Prathap Jayaram: https://orcid.org/0000-0001-62095410; Wonjae Lee: https://orcid.org/0000-0001-6028-3565; Michael Y. Lee: https:// orcid.org/0000-0001-5240-9885

\section{Author Contributions}

Conceptualization: Lee JH, Lee W, Lee MY. Data acquisition: Lee JH, Choi EJ, Han SC, Chung HS, Lee W. Data analysis or interpretation: Kwon MJ, Jayaram P, Lee W, Lee MY. Drafting of the manuscript: Lee JH, Choi EJ, Han SC, Chung HS, Kwon MJ, Jayaram P, Lee W, Lee MY. Critical revision of the manuscript: Kwon MJ, Lee W, Lee MY. Approval of the final version of the manuscript: all authors.

\section{Conflict of Interest}

No potential conflict of interest relevant to this article was reported.

\section{References}

1. Fields BK, Skalski MR, Patel DB, White EA, Tomasian A, Gross JS, et al. Adhesive capsulitis: review of imaging findings, pathophysiology, clinical presentation, and treatment options. Skeletal Radiol 2019;48:1171-1184.

2. Manske RC, Prohaska D. Diagnosis and management of adhesive capsulitis. Curr Rev Musculoskelet Med 2008;1:180-189.

3. Huang SW, Lin JW, Wang WT, Wu CW, Liou TH, Lin HW. Hyperthyroidism is a risk factor for developing adhesive capsulitis of the shoulder: a nationwide longitudinal population-based study. Sci Rep 2014;4:4183.

4. Ahn JH, Lee DH, Kang H, Lee MY, Kang DR, Yoon SH. Early intraarticular corticosteroid injection improves pain and function in adhesive capsulitis of the shoulder: 1-year retrospective longitudinal study. PM R 2018;10:19-27.

5. Shaffer B, Tibone JE, Kerlan RK. Frozen shoulder: a long-term follow-up. J Bone Joint Surg Am 1992;74:738-746. 
6. Grey RG. The natural history of "idiopathic" frozen shoulder. J Bone Joint Surg Am 1978;60:564.

7. Harrast MA, Rao AG. The stiff shoulder. Phys Med Rehabil Clin N Am 2004;15:557-573.

8. Lee SY, Lee KJ, Kim W, Chung SG. Relationships between capsular stiffness and clinical features in adhesive capsulitis of the shoulder. PM R 2015;7:1226-1234.

9. Le HV, Lee SJ, Nazarian A, Rodriguez EK. Adhesive capsulitis of the shoulder: review of pathophysiology and current clinical treatments. Shoulder Elbow 2017;9:75-84.

10. Dupeyron A, Denarie M, Richard D, Dobija L, Castelli C, Petiot S, et al. Analgesic gas for rehabilitation of frozen shoulder: protocol for a randomized controlled trial. Ann Phys Rehabil Med 2019;62:4348.

11. Scarlat MM. Complications with reverse total shoulder arthroplasty and recent evolutions. Int Orthop 2013;37:843-851.

12. Lorbach O, Anagnostakos K, Scherf C, Seil R, Kohn D, Pape D. Nonoperative management of adhesive capsulitis of the shoulder: oral cortisone application versus intra-articular cortisone injections. J Shoulder Elbow Surg 2010;19:172-179.

13. Wu WT, Chang KV, Han DS, Chang CH, Yang FS, Lin CP. Effectiveness of glenohumeral joint dilatation for treatment of frozen shoulder: a systematic review and meta-analysis of randomized controlled trials. Sci Rep 2017;7:10507.

14. Buhren BA, Schrumpf $H$, Hoff NP, Bolke E, Hilton S, Gerber PA. Hyaluronidase: from clinical applications to molecular and cellular mechanisms. Eur J Med Res 2016;21:5.

15. Ruschen $H$, Aravinth K, Bunce C, Bokre D. Use of hyaluronidase as an adjunct to local anaesthetic eye blocks to reduce intraoperative pain in adults. Cochrane Database Syst Rev 2018;3:CD010368.

16. Byun SD, Park DH, Hong YH, Lee ZI. The additive effects of hyaluronidase in subacromial bursa injections administered to patients with peri-articular shoulder disorder. Ann Rehabil Med 2012;36:105-111.

17. Kim SB, Lee KW, Lee JH, Kim MA, An BW. The effect of hyaluronidase in interlaminar lumbar epidural injection for failed back surgery syndrome. Ann Rehabil Med 2012;36:466-473.

18. Choi WD, Cho DH, Hong YH, Noh JH, Lee ZI, Byun SD. Effects of subacromial bursa injection with corticosteroid and hyaluronidase according to dosage. Ann Rehabil Med 2013;37:668-674.

19. Allen GM. Shoulder ultrasound imaging-integrating anatomy, biomechanics and disease processes. Eur J Radiol 2008;68:137146.

20. Tandon A, Dewan S, Bhatt $S$, Jain AK, Kumari R. Sonography in diagnosis of adhesive capsulitis of the shoulder: a case-control study. J Ultrasound 2017;20:227-236.

21. Ryan V, Brown H, Minns Lowe CJ, Lewis JS. The pathophysiology associated with primary (idiopathic) frozen shoulder: a systematic review. BMC Musculoskelet Disord 2016;17:340.
22. Corazza A, Orlandi D, Fabbro E, Ferrero G, Messina C, Sartoris R, et al. Dynamic high-resolution ultrasound of the shoulder: how we do it. Eur J Radiol 2015;84:266-277.

23. Ryans I, Montgomery A, Galway R, Kernohan WG, McKane R. A randomized controlled trial of intra-articular triamcinolone and/ or physiotherapy in shoulder capsulitis. Rheumatology (Oxford) 2005;44:529-535.

24. Cho CH, Song KS, Kim BS, Kim DH, Lho YM. Biological aspect of pathophysiology for frozen shoulder. Biomed Res Int 2018;2018:7274517.

25. van der Windt DA, Koes BW, Deville W, Boeke AJ, de Jong BA, Bouter LM. Effectiveness of corticosteroid injections versus physiotherapy for treatment of painful stiff shoulder in primary care: randomised trial. BMJ 1998;317:1292-1296.

26. Stout A, Friedly J, Standaert CJ. Systemic absorption and side effects of locally injected glucocorticoids. PM R 2019;11:409-419.

27. Pogorzelski J, Imhoff AB, Degenhardt H, Siebenlist S. Primary (idiopathic) shoulder stiffness: definition, disease progression, epidemiology and etiology. Unfallchirurg 2019;122:917-924.

28. Alsubheen SA, Nazari G, Bobos P, MacDermid JC, Overend TJ, Faber $K$. Effectiveness of nonsurgical interventions for managing adhesive capsulitis in patients with diabetes: a systematic review. Arch Phys Med Rehabil 2019;100:350-365.

29. Habib G, Sakas F, Artul S, Khazin F, Hakim G, Jabbour A, et al. The impact of intra-articular methylprednisolone acetate injection on fructosamine levels in diabetic patients with osteoarthritis of the knee, a case-control study. Clin Rheumatol 2016;35:1609-1614.

30. Kim YS, Lee HJ, Lee DH, Choi KY. Comparison of high- and lowdose intra-articular triamcinolone acetonide injection for treatment of primary shoulder stiffness: a prospective randomized trial. J Shoulder Elbow Surg 2017;26:209-215.

31. Kim KH, Park JW, Kim SJ. High- vs low-dose corticosteroid injection in the treatment of adhesive capsulitis with severe pain: a randomized controlled double-blind study. Pain Med 2018;19:735741.

32. Simoni P, Grumolato M, Malaise O, Preziosi M, Pasleau F, de Lemos Esteves F. Are blind injections of gleno-humeral joint (GHJ) really less accurate imaging-guided injections? A narrative systematic review considering multiple anatomical approaches. Radiol Med 2017;122:656-675.

33. Ikeda K. Roles of musculoskeletal ultrasonography in the management of rheumatic diseases. Rinsho Byori 2015;63:580589.

34. Xiao RC, Walley KC, DeAngelis JP, Ramappa AJ. Corticosteroid Injections for adhesive capsulitis: a review. Clin J Sport Med 2017;27:308-320.

35. Bloom JE, Rischin A, Johnston RV, Buchbinder R. Image-guided versus blind glucocorticoid injection for shoulder pain. Cochrane Database Syst Rev 2012;(8):CD009147. 